\title{
The chiral condensate in neutron matter *
}

\author{
T. Krüger ${ }^{1,2}$, I. Tews ${ }^{1,2}$, B. Friman ${ }^{3}$, K. Hebeler ${ }^{1,2}$, and A. Schwenk ${ }^{2,1}$ \\ ${ }^{1}$ IKP, Technische Universität Darmstadt, Darmstadt, Germany; ${ }^{2}$ EMMI, GSI, Darmstadt, Germany; ${ }^{3}$ GSI, Darmstadt, \\ Germany
}

The chiral condensate is an order parameter for characterizing the chiral phase transition in dense and hot strongly interacting matter. Owing to the fermion sign problem, there are no first-principle QCD results for the phase diagram at low temperatures and high densities, the conditions probed in neutron stars. Recent observations of neutron stars with $2 M_{\odot}$ masses provide general constraints on the equation of state (EOS) of cold strongly interacting matter, and put into question whether exotic phases that tend to soften the EOS are realized in neutron stars. At densities $n \lesssim n_{0}$, where $n_{0}=0.16 \mathrm{fm}^{-3}$ denotes nuclear saturation density, the properties of nuclear systems have been studied systematically based on nuclear forces derived within chiral effective field theory and using renormalization group methods. In this work, we use chiral EFT interactions to study the chiral condensate as a function of density in neutron matter, based on perturbative calculations around the first-order Hartree-Fock energy.

The chiral condensate in neutron matter relative to the vacuum is given by [1]

$$
\begin{aligned}
\frac{\langle\bar{q} q\rangle_{n}}{\langle\bar{q} q\rangle_{0}}= & 1-\frac{n}{f_{\pi}^{2}} \frac{\sigma_{\pi N}}{m_{\pi}^{2}}\left(1-\frac{3 k_{F}^{2}}{10 m_{N}^{2}}+\ldots\right) \\
& -\frac{n}{f_{\pi}^{2}} \frac{\partial}{\partial m_{\pi}^{2}} \frac{E_{\text {int }}\left(m_{\pi}, k_{F}\right)}{N} .
\end{aligned}
$$

The leading $\sigma_{\pi N}$ contribution to the chiral condensate in Eq. (1), which is due to the mass term in $E_{\text {free }} / N$, is linear in density and is shown in Fig. 1 by the dashed line. For the density range shown in Fig. 1, where chiral EFT interactions can be applied with confidence, the kinetic energy contribution is only a $4 \%$ correction relative to the leading term, while relativistic corrections, indicated by the dots in Eq. (1), are negligible at these densities [1].

We calculate the explicit $m_{\pi}$ dependence of nuclear forces by varying the value of the pion mass in the pionexchange $\mathrm{NN}, 3 \mathrm{~N}$, and $4 \mathrm{~N}$ interactions. At the $\mathrm{NN}$ level, we use the $\mathrm{N}^{3} \mathrm{LO}$ potentials of Epelbaum, Glöckle, and Meißner [2] with cutoffs $450 / 500$ and $450 / 700 \mathrm{MeV}$. With these $\mathrm{NN}$ interactions neutron matter is perturbative at the densities considered here [3, 4].

We find that nuclear interactions impede the restoration of chiral symmetry in neutron matter at zero temperature. The net effect of interactions remains below $10 \%$ for $n \lesssim 0.2 \mathrm{fm}^{-3}$, but grows with increasing density. The dominant source of uncertainty is the $\sigma_{\pi N}$ term. We conclude that for moderate densities, say $n \lesssim 0.3 \mathrm{fm}^{-3}$, a chi-

*Work supported in part by EMMI, by the ERC Grant No. 307986 STRONGINT and by the DFG through SFB 634.

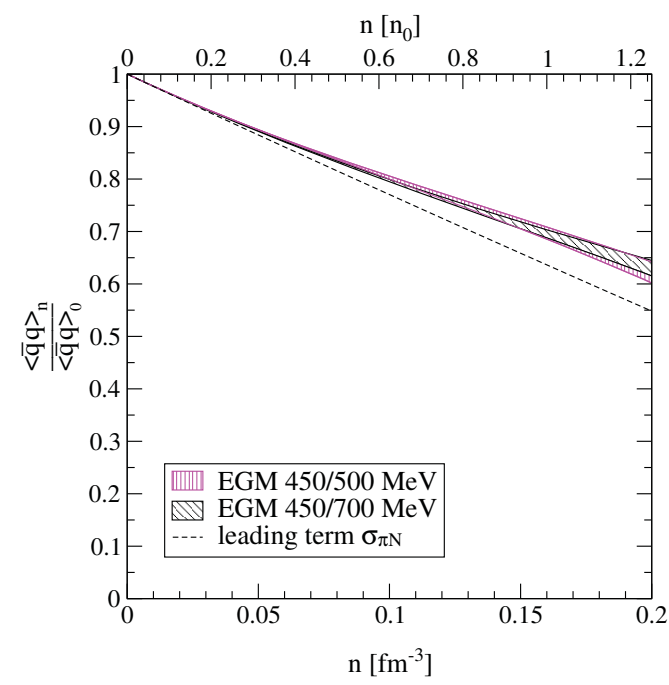

Figure 1: Chiral condensate $\langle\bar{q} q\rangle_{n} /\langle\bar{q} q\rangle_{0}$ as a function of density in neutron matter [1]. The dashed line is the leading pion-nucleon sigma-term contribution. The interaction contributions are obtained from the $\mathrm{N}^{3} \mathrm{LO}$ neutron-matter calculation of Refs. [3, 4]. The bands for each NN potential include uncertainties of the many-body calculation, of the $c_{i}$ couplings of $3 \mathrm{~N}$ forces, and those resulting from the $3 \mathrm{~N} / 4 \mathrm{~N}$ cutoff variation.

ral phase transition in neutron-rich matter therefore seems unlikely, although we cannot exclude a strong first-order transition. For the densities considered here, we find a good convergence of the chiral condensate from $\mathrm{N}^{2} \mathrm{LO}$ to $\mathrm{N}^{3} \mathrm{LO}$ in chiral EFT. It would be very interesting to calculate the chiral condensate also for higher densities. While a systematic calculation in chiral EFT is difficult at densities much higher than $n=0.2 \mathrm{fm}^{-3}$, astrophysical observations provide valuable constraints.

\section{References}

[1] T. Krüger, I. Tews, B. Friman, K. Hebeler, A. Schwenk, Phys. Lett. B276, 412 (2013).

[2] E. Epelbaum, W. Glöckle, and Ulf-G. Meißner, Eur. Phys. J. A 19, 401 (2004); Nucl. Phys. A 747, 362 (2005).

[3] I. Tews, T. Krüger, K. Hebeler, and A. Schwenk, Phys. Rev. Lett. 110, 032504 (2013).

[4] T. Krüger, I. Tews, K. Hebeler, and A. Schwenk, Phys.Rev. C88, 025802 (2013). 